\title{
Relation of the fourth-order statistical invariants of velocity gradient tensor in isotropic turbulence
}

\author{
L. Fang, ${ }^{1}$ Y. J. Zhang, ${ }^{1}$ J. Fang,,${ }^{2,3,{ }^{*}}$ and Y. Zhu ${ }^{4}$ \\ ${ }^{1}$ LMP, Ecole Centrale de Pékin, Beihang University, Beijing 100191, China \\ ${ }^{2}$ National Key Laboratory of Science and Technology on Aero-Engine Aero-Thermodynamics, School of Energy and Power Engineering, \\ Beihang University, Beijing 100191, China \\ ${ }^{3}$ Computer Science and Engineering Department, STFC Daresbury Laboratory, Warrington WA4 4AD, United Kingdom \\ ${ }^{4}$ LMFA, Ecole Centrale de Lyon, Université de Lyon, 69130 Ecully, France
}

(Received 31 May 2016; published 30 August 2016)

\begin{abstract}
We show by direct numerical simulations (DNSs) that in different types of isotropic turbulence, the fourth-order statistical invariants have approximately a linear relation, which can be represented by a straight line in the phase plane, passing two extreme states: the Gaussian state and the restricted Euler state. Also, each DNS case corresponds to an equilibrium region that is roughly Reynolds-dependent. In addition, both the time reversal and the compressibility effect lead to nonequilibrium transition processes in this phase plane. This observation adds a new restriction on the mean-field theory.
\end{abstract}

DOI: 10.1103/PhysRevE.94.023114

\section{INTRODUCTION}

In homogeneous isotropic turbulence (HIT), the expression of the statistical moments of a velocity gradient tensor has always been considered an important basic problem [1]. The quasi-Gaussian closures [2,3] assume a relation between the second- and fourth-order moments, while the direct interaction approximation (DIA) relaxes this restriction [4] and captures more details on the fourth-order accumulations [5,6]. For all of these existing closure methods, the high-order moments are always difficult problems. In fact, there are even quite a few studies on understanding and explaining the high-order statistics.

It is not difficult to show that both the second-order [7] and third-order moments [8] of a velocity gradient tensor have only one degree of freedom, but for the fourth-order moments it is more complicated. From Siggia [9] one can obtain four invariants, which are expressed by the combinations of vorticity and strain rate. Several studies have been performed to deduce the exact formation of the fourth-order moments [9-11]. In particular, from Hierro and Dopazo [11] these four invariants can be defined as

$$
F_{1}=\left\langle u_{1,1}^{4}\right\rangle, F_{2}=\left\langle u_{1,1}^{2} u_{2,1}^{2}\right\rangle, F_{3}=\left\langle u_{2,1}^{4}\right\rangle, F_{4}=\left\langle u_{1,1}^{2} u_{2,3}^{2}\right\rangle,
$$

with \langle\rangle the ensemble average and $u_{i, j}=\partial u_{i} / \partial x_{j}$ the velocity gradient tensor. In some of the literature, these four invariants are alternatively written in the formula

$$
I_{1}=\left\langle s^{4}\right\rangle, I_{2}=\left\langle s^{2} \omega^{2}\right\rangle, I_{3}=\left\langle\omega_{i} s_{i j} \omega_{k} s_{k j}\right\rangle, I_{4}=\left\langle\omega^{4}\right\rangle,
$$

with $s^{2}=s_{i j} s_{i j}, s_{i j}=\left(\partial u_{i} / \partial x_{j}+\partial u_{j} / \partial x_{i}\right) / 2$, and $\omega$ is the vorticity. The transformation between (1) and (2) can be found in Ref. [11].

In the present contribution, we aim to investigate the relation among these invariants in real turbulence. We show by different types of direct numerical simulation (DNS) cases that the locations of $\left(F_{2} / F_{1}, F_{3} / F_{1}\right)$ in the phase plane are always

\footnotetext{
*fangjian19@gmail.com
}

near a straight line, which can be defined by two extreme theoretical states. In addition, we show the evolution trends in this phase plane for compressible turbulence and timereversed nonequilibrium turbulence. Finally, the observation is employed in the mean-field theory to provide a new restriction.

\section{EXTREME STATES}

In this section, we list two typical extreme states that were employed in turbulence closures, and we show that both states imply typical constant points in the $\left(F_{2} / F_{1}, F_{3} / F_{1}\right)$ phase plane.

\section{A. Gaussian state}

The quasi-Gaussian assumption is widely used in turbulence closures such as the eddy-damping quasinormal Markovian (EDQNM) theory [2]. Similar to that, we may obtain Gaussian relations in the physical space for the fourth-order moments in Eq. (1), which simply leads to

$$
\begin{aligned}
& F_{1}=\left\langle u_{1,1}^{4}\right\rangle=3\left\langle u_{1,1}^{2}\right\rangle\left\langle u_{1,1}^{2}\right\rangle, \\
& F_{2}=\left\langle u_{1,1}^{2} u_{2,1}^{2}\right\rangle=\left\langle u_{1,1}^{2}\right\rangle\left\langle u_{2,1}^{2}\right\rangle, \\
& F_{3}=\left\langle u_{2,1}^{4}\right\rangle=3\left\langle u_{2,1}^{2}\right\rangle\left\langle u_{2,1}^{2}\right\rangle, \\
& F_{4}=\left\langle u_{1,1}^{2} u_{2,3}^{2}\right\rangle=\left\langle u_{1,1}^{2}\right\rangle\left\langle u_{2,1}^{2}\right\rangle .
\end{aligned}
$$

Considering the relation in HIT that $\left\langle u_{1,1}^{2}\right\rangle=\frac{1}{2}\left\langle u_{2,1}^{2}\right\rangle$ [12], we have finally

$$
F_{1}: F_{2}: F_{3}: F_{4}=1: \frac{2}{3}: 4: \frac{2}{3},
$$

or using the other invariants,

$$
I_{1}: I_{2}: I_{3}: I_{4}=1: \frac{10}{7}: \frac{10}{21}: \frac{100}{21} .
$$

\section{B. Restricted Euler state}

Another extreme state, known as the restricted Euler (RE) state, corresponds to vanishing pressure and viscous terms in the Navier-Stokes equations and retaining the advection 
term [13]. As discussed by Hill and Boratav, the RE state yields [see Eqs. (12) and (13) of Ref. [14]]

$$
\begin{aligned}
\partial_{r} D_{1111}(r)+\frac{2}{r}\left[D_{1111}(r)-3 D_{11 \alpha \alpha}(r)\right] & =0, \\
\partial_{r} D_{11 \alpha \alpha}(r)-\frac{4}{3 r}\left[D_{\alpha \alpha \alpha \alpha}(r)-3 D_{11 \alpha \alpha}(r)\right] & =0,
\end{aligned}
$$

where $D$ is the fourth-order structure function with the two-point distance $r$, and the subscripts 1 and $\alpha$ denote the longitudinal and transverse components, respectively (without taking summation). Taking the limit of Eq. (6) when $r \rightarrow 0$, one obtains finally

$$
F_{1}: F_{2}: F_{3}=1: \frac{1}{2}: \frac{15}{8} .
$$

The value of $F_{4}$ is independent of the other three values in the RE state. Due to the uncertainty of $F_{4}$, we cannot obtain any exact relation on the invariants $I_{1}, \ldots, I_{4}$.

We remark that although the RE system leads to singularity of the velocity gradient in finite time [13], the RE simplification provides an analytical tool to explain the invariants that can be nonsingular, for example the so-called $Q-R$ phase-space problem [15-18]. $Q$ and $R$ are defined as second- and thirdorder invariants, respectively, while in the present contribution we show similarly that the RE system leads to an extreme state for the fourth-order invariants.

Comparing the Gaussian state and the RE state, it is possible to define an $\left(F_{2} / F_{1}, F_{3} / F_{1}\right)$ phase plane, in which the two states correspond to two typical points. In the following section, we will show by DNS databases the behavior of real turbulence in this phase plane.

\section{NUMERICAL RESULTS}

In this section, we summarize and perform DNS for different types of HIT flows to investigate the statistical behavior in the $\left(F_{2} / F_{1}, F_{3} / F_{1}\right)$ phase plane, including forced incompressible turbulence, free-decaying and time-reversed nonequilibrium incompressible turbulence, and compressible turbulence.

\section{A. Forced incompressible turbulent flows}

In the work of Ishihara et al. [19], the fourth-order statistical moments were carefully examined with high-resolution DNS by using the invariants $I_{1}, \ldots, I_{4}$, and they were discussed together with the DNS data by Kerr [20]. We calculate the values of $F_{2} / F_{1}$ and $F_{3} / F_{1}$ from these data and plot them in Fig. 1. It is obvious that all the points are located around the straight line passing both the Gaussian state and the RE state (denoted as the "Gaussian-RE line" in the present contribution), which implies that each field can be statistically regarded as a superposition of these two extreme states. Indeed, this is because each invariant $F_{i}$ is a different mix of the invariants $I_{1}, \ldots, I_{4}$, which are roughly of the same scaling in Ref. [19], expect for lower Reynolds numbers. Another observed fact is the trend in each group that when the Reynolds number decreases, the field usually moves toward the Gaussian state in the $\left(F_{2} / F_{1}, F_{3} / F_{1}\right)$ phase plane (except for several cases). This may be because in lower-Reynolds turbulence the

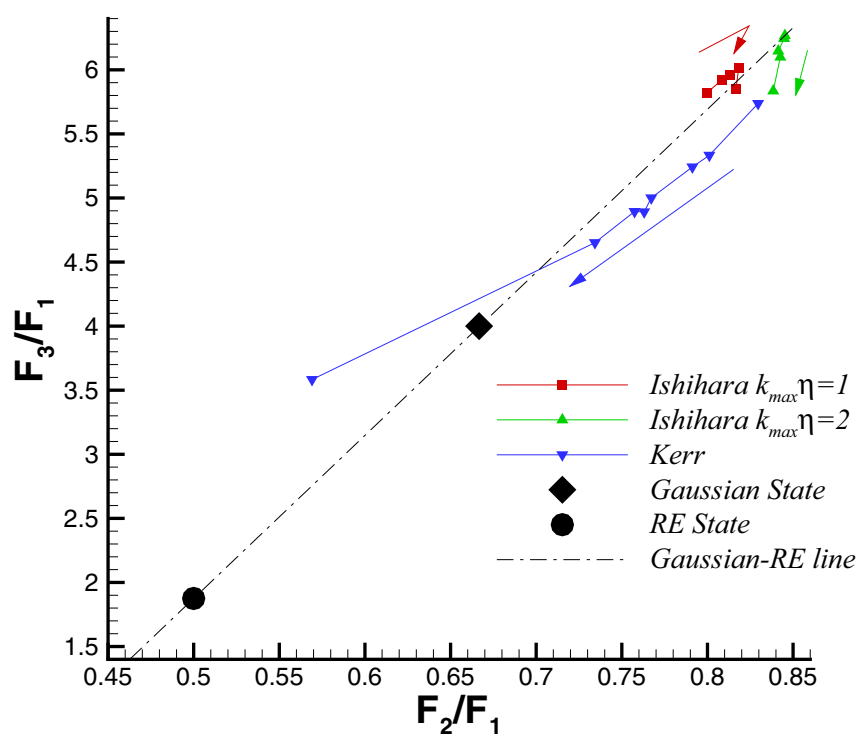

FIG. 1. Comparisons of $F_{2} / F_{1}$ and $F_{3} / F_{1}$ in forced incompressible turbulent flows. $12<\operatorname{Re}_{\lambda}<1133$. The arrows denote the decrease of the Reynolds number in each group of DNS cases.

inertial range is shorter, and the dissipative range, which is close to a Gaussian state, occupies more parts in the scale space. Therefore, phenomenally we may conclude that when a turbulent flow is more "well-organized," it is located farther away from the Gaussian state in the $\left(F_{2} / F_{1}, F_{3} / F_{1}\right)$ phase plane with greater values of $F_{2} / F_{1}$ and $F_{3} / F_{1}$ (we call this "over-Gaussian" in the present contribution).

\section{B. Free-decaying and time-reversed nonequilibrium incompressible turbulent flows}

We perform a series of DNS runs with $384^{3}$ resolution with regard to the free-decaying turbulence as well its time-reversed nonequilibrium cases. We review here that the idea of time reversal is to construct a gedanken experiment [21], which leads to extreme spectrally nonequilibrium turbulent flows [22]. The numerical details of the calculation method can be found in Refs. [23] and [21]. A free-decaying turbulence is calculated and is denoted as "Normal-Normal" since all scales are normally decaying; two parallel nonequilibrium cases are performed by reversing the velocity field of the "Normal-Normal" case (with "Reverse-Normal" reversing only the large-scale field, while "Reverse-Reverse" reverses all scales). The Reynolds number is $\operatorname{Re}_{\lambda}=80.8$ at the time of reversal. From Fig. 2(a) it is clear that all cases are located near the Gaussian-RE line in the over-Gaussian region, which is consistent with the results of forced turbulence as discussed in the previous subsection. In Fig. 2(b) it is shown that the free-decaying case is located in a small statistically equilibrium region with $0.80<F_{2} / F_{1}<0.83$, while the other nonequilibrium cases correspond to increasing values of $F_{2} / F_{1}$ and $F_{3} / F_{1}$ in a short time (i.e., in about one turnover time) and statistically equilibrium values (about $0.825<F_{2} / F_{1}<$ $0.855)$ in a longer time. Considering the analysis in the previous subsection, this short-time nonequilibrium transition procedure can be considered as a self-organizing process, 


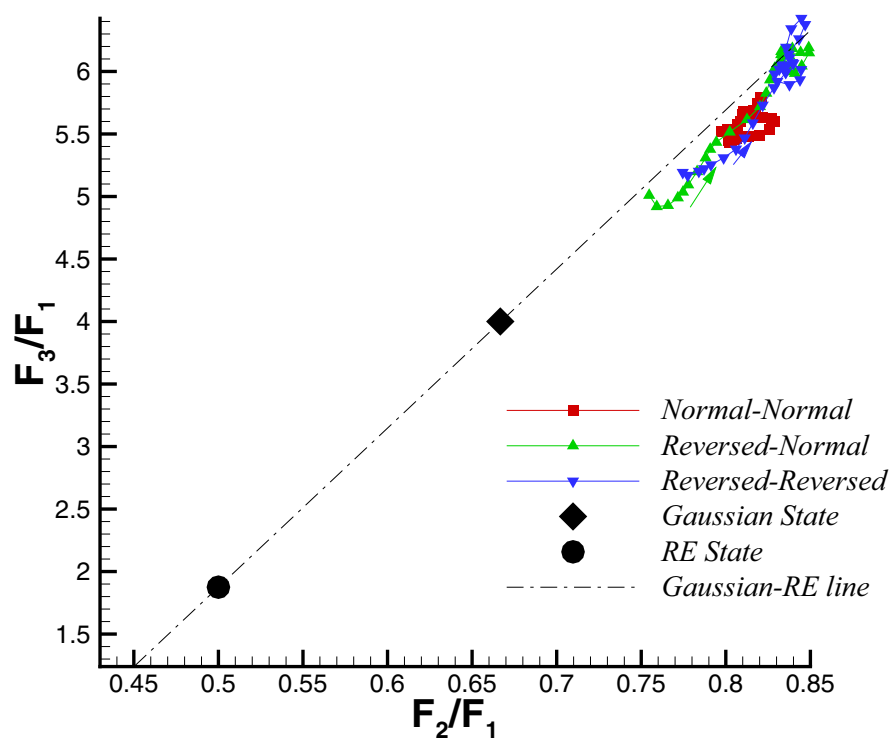

(a)

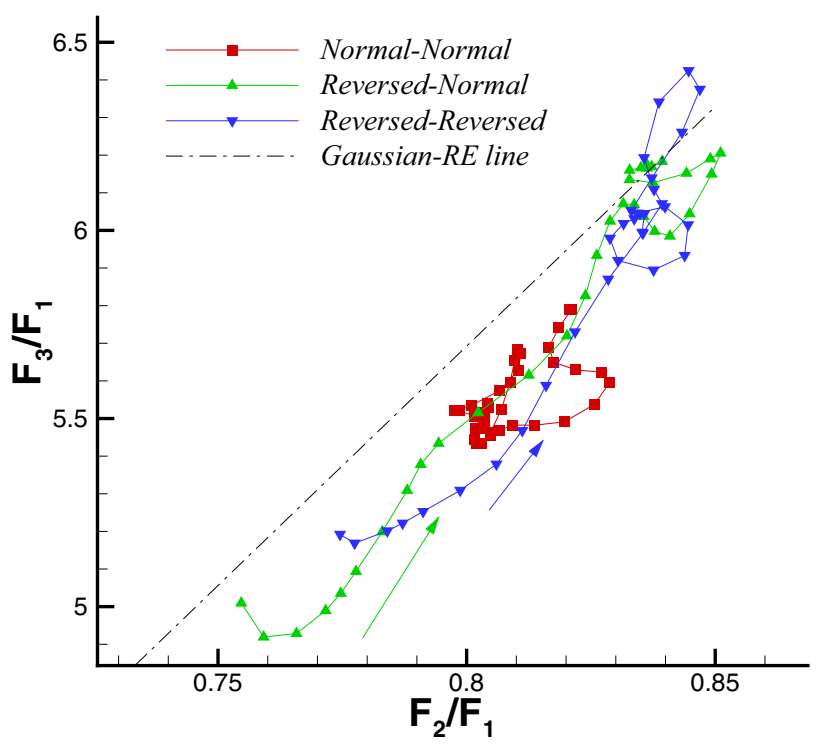

(b)

FIG. 2. Comparisons of $F_{2} / F_{1}$ and $F_{3} / F_{1}$ in free-decaying turbulence (denoted as "Normal-Normal" in the figure) and time-reversed nonequilibrium turbulent flows (with "Reverse-Normal" reversing only the large-scale field, while "Reverse-Reverse" reversing all scales). $\operatorname{Re}_{\lambda}=80.8$ at the time of reversal. The arrows denote the direction of time in each group of DNS cases. (a) Global view. (b) Zoomed view.

which is consistent with our a priori tests for other statistical quantities such as the skewness of the velocity gradient [22].

\section{Free-decaying compressible turbulent flows}

To the best of our knowledge, there is still no existing research on the effect of compressibility on the fourth-order moments of a velocity gradient. Here we perform a series of DNS cases to investigate the behavior of compressible turbulence. The nondimensional, compressible Navier-Stokes equations in the strong conservation form are solved within the framework of a high-order finite-difference method. A seventhorder low-dissipative monotonicity-preserving scheme [24] is adopted to capture shock waves that emerge from the strong compressible turbulence, while a classic sixth-order compact central scheme is used to solve the viscous terms [25]. The Navier-Stokes equations are advanced in time by using the third-order total variation diminishing Runge-Kutta scheme [26]. The periodic boundary conditions are employed in all three directions [27]. The initial thermodynamic variables have uniform distributions, and the initial velocity field is generated according to the method of Rogallo [28] and satisfies the Gaussian spectral density function. A $512^{3}$ resolution is employed with initial Reynolds number $\operatorname{Re}_{\lambda}=72$ and initial turbulent Mach numbers $\mathrm{Ma}_{0}=0.1,0.3,0.5,0.7,0.9$, and 1.2, respectively. Figure 3(a) shows the fourth-order behavior with low initial Mach numbers $\left(\mathrm{Ma}_{0} \leqslant 0.5\right)$, while Fig. 3(b) shows the behavior with higher initial Mach numbers $\left(\mathrm{Ma}_{0} \geqslant 0.7\right)$. Similar to the results in the previous subsections, all points are also located near the Gaussian-RE line. The difference is that here, the initial fields of these compressible cases are very distinct in the $\left(F_{2} / F_{1}, F_{3} / F_{1}\right)$ phase plane: higher initial Mach numbers yield greater values of $F_{2} / F_{1}$ and $F_{3} / F_{1}$. For the $\mathrm{Ma}_{0}=0.7$ case, the initial field is near the RE state, while for the $\mathrm{Ma}_{0}=0.9$ and 1.1 cases, the initial fields are located at the other side with small $F_{2} / F_{1}$ and $F_{3} / F_{1}$ in the phase plane (we call it "over-RE" by comparing to the over-Gaussian states). Another fact observed in the figures is that after a transition process, all cases reach their own equilibrium status. The difference is that for small initial Mach numbers, the transition processes are not monotonous, as shown in Fig. 3(a). This transition process in the $\left(F_{2} / F_{1}, F_{3} / F_{1}\right)$ phase plane may imply new physics, since traditional investigations of low-order statistics of free-decaying compressible turbulence do not indicate such a transition. As discussed in previous subsections, this transition implies a self-organizing process for the fourth-order statistics.

\section{DISCUSSIONS}

\section{A. Gaussian-RE line and pressure effect}

It is quite difficult to systematically explain the GaussianRE relation using a complete theory. However, at least we can say that this phenomenon is closely related to the pressure effect. This can be explained by comparing to the RE simplification, where the anisotropic pressure Hessian is omitted. If we reconsider this pressure Hessian and obtain the statistically steady Navier-Stokes equations, we have [by taking the limit of $r \rightarrow 0$ in Eqs. (5) and (6) of Ref. [14]]

$$
\begin{aligned}
F_{1}-2 F_{2} & =-\frac{1}{\rho}\left\langle u_{1,1}^{2} p_{, 11}\right\rangle, \\
5 F_{2}-\frac{4}{3} F_{3} & =-\frac{1}{\rho}\left\langle u_{2,1}^{2} p_{, 11}+2 u_{1,1} u_{2,1} p_{, 12}\right\rangle,
\end{aligned}
$$

with $p$ pressure and $p_{, i j}=\partial^{2} p / \partial x_{i} \partial x_{j}$.

The right-hand-side terms of Eq. (8) represent the correlations between the pressure Hessian in RE and the velocity gradients. Clearly, these correlations lead to the departure of the RE state in the $\left(F_{2} / F_{1}, F_{3} / F_{1}\right)$ plane. These pressure- 


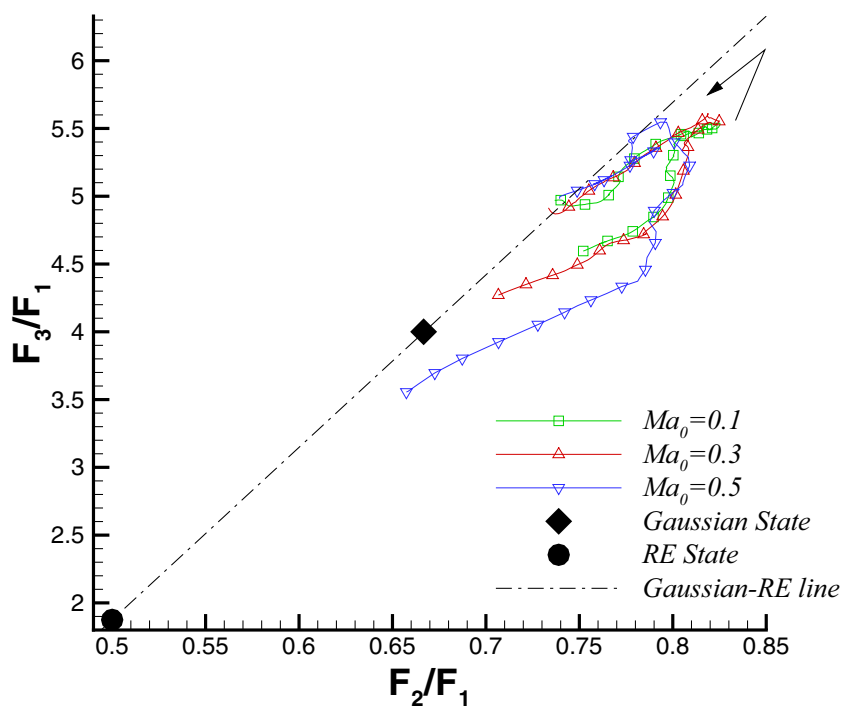

(a)

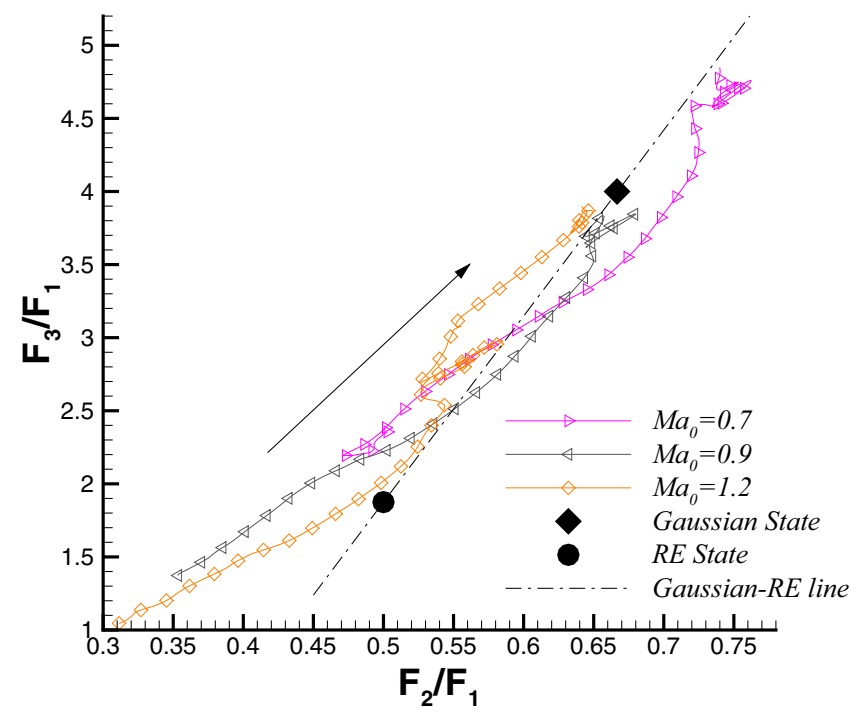

(b)

FIG. 3. Comparisons of $F_{2} / F_{1}$ and $F_{3} / F_{1}$ in free-decaying compressible flows. The arrows denote the direction of time in each group of DNS cases. $14<\operatorname{Re}_{\lambda}<70$. (a) $\mathrm{Ma}_{0} \leqslant 0.5 ;$ (b) $\mathrm{Ma}_{0} \geqslant 0.7$.

velocity gradient correlations are indeed central problems of the RE system, which have attracted many studies, including geometrical considerations [29,30], assumptions on the short-time deformation [31], and assumptions on the Gaussianity of the pressure Hessian [32]; however, presently the understanding is still not clear. From the present study, the Gaussian-RE line simply implies an approximate relation

$$
\left\langle 6 u_{1,1}^{2} p_{, 11}-u_{2,1}^{2} p_{, 11}-2 u_{1,1} u_{2,1} p_{, 12}\right\rangle=0,
$$

which is expected to inspire future research on the pressurevelocity gradient correlations.

We recall another fact to explain and to support the Gaussian-RE line phenomenon. In the investigation of the short-term evolution of Lagrangian velocity gradient correlation, it is found that for statistically steady turbulence, there should be (see Sec. III A of Ref. [33])

$$
\left\langle\dot{u}_{i, j} \dot{u}_{j, i}\right\rangle /\left\langle\dot{u}_{i, j} \dot{u}_{i, j}\right\rangle \approx 0.6,
$$

where $\boldsymbol{b}$ is the Lagrangian derivative. Without omitting the pressure term or the viscosity term, the incompressible NavierStokes equations lead to [see Eq. (4) of Ref. [13]]

$$
\dot{u}_{i, j}=-u_{i, k} u_{k, j}-\frac{1}{\rho} p_{, i j}+v u_{i, j k k} .
$$

Substituting Eq. (11) into (10) yields the following formula:

$$
\frac{\left\langle u_{i, k} u_{k, j} u_{j, m} u_{m, i}\right\rangle+\frac{2}{\rho}\left\langle u_{i, k} u_{k, j} p_{, i j}\right\rangle+\cdots}{\left\langle u_{i, k} u_{k, j} u_{i, m} u_{m, j}\right\rangle+\frac{2}{\rho}\left\langle u_{i, k} u_{k, j} p_{, i j}\right\rangle+\cdots} \approx 0.6 .
$$

We do not write all terms here, but only emphasize that some pressure-related terms are symmetric to the subscripts and therefore remain the same between numerator and denominator [e.g., $\left\langle u_{i, k} u_{k, j} p_{, i j}\right\rangle$, corresponding to the right-hand side of Eq. (8)], while some terms are not (e.g., the fourthorder velocity gradient correlations $\left\langle u_{i, k} u_{k, j} u_{j, m} u_{m, i}\right\rangle$ and $\left\langle u_{i, k} u_{k, j} u_{i, m} u_{m, j}\right\rangle$, which can be decomposed to the invariants $\left.F_{1}, \ldots, F_{4}\right)$. The ratio 0.6 represents a balance among them that is roughly Reynolds-independent [33]. This is quite similar to our discussion above, i.e., Eqs. (8) and (9), although many other additional terms also appear here in Eq. (12). In brief, though not rigorously, the constant 0.6 implies that there is a Reynolds-independent relation for the pressure effect of the fourth-order velocity gradient correlations, which is consistent with the Gaussian-RE result (9) and therefore supports the present work.

In addition, the studies of Yeung et al. $[34,35]$ might also be related to the present contribution, and they show approximate linearity between the high-order vorticity flatness and the high-order strain flatness. Although the underlying reason is not clear, this fact might also be useful evidence for future investigations of the constraints of high-order statistics in turbulent flows.

\section{B. Using the Gaussian-RE line in the mean-field theory}

As shown previously from the DNS databases, the Gaussian-RE line might be a good approximation for varies turbulent fields. This fact involves a new restriction that can be employed in statistical turbulence models concerning fourth-order statistics, such as, for instance, the mean-field theory (MFT).

The MFT was introduced to take into account the neglected pressure terms of the RE state $[14,36]$. It was found that neglecting the pressure term involves an imbalance of the approximate equation for the fourth-order structure function at the inertial range, of the order of $10 \%$ [36]. In MFT, two underdetermined functions $G$ and $H$ were observed to be constant in the inertial range, but in the dissipative range this is not the case. Similar to the discussion in the previous section, 
we have

$$
\begin{aligned}
\partial_{r} D_{1111}(r)+\frac{1}{r} & {\left[(2+3 G) D_{1111}(r)\right.} \\
& \left.-\left(6+3 G \frac{D_{11}(r)}{D_{\alpha \alpha}(r)}\right) D_{11 \alpha \alpha}(r)\right]=0 \\
\partial_{r} D_{11 \alpha \alpha}(r)+\frac{1}{r} & {\left[(4+G+2 H) D_{11 \alpha \alpha}(r)\right.} \\
& -\left(\frac{4}{3}+G \frac{D_{11}(r)}{D_{\alpha \alpha}(r)}\right) D_{\alpha \alpha \alpha \alpha}(r) \\
& \left.-2 H \frac{D_{1 \alpha \alpha}^{2}(r)}{D_{\alpha \alpha}(r)}\right]=0 .
\end{aligned}
$$

For the third-order moments, in HIT we have

$$
\begin{aligned}
\lim _{r \rightarrow 0} \frac{1}{r^{4}} \frac{D_{1 \alpha \alpha}^{2}(r)}{D_{\alpha \alpha}(r)} & =\frac{\left\langle u_{1,1} u_{2,1}^{2}\right\rangle^{2}}{\left\langle u_{2,1}^{2}\right\rangle}=\frac{\frac{4}{9}\left\langle u_{1,1}^{3}\right\rangle^{2}}{2\left\langle u_{1,1}^{2}\right\rangle} \\
& =\frac{2}{9} S^{2}\left\langle u_{1,1}^{2}\right\rangle^{2}=\frac{2}{9} \frac{S^{2}}{K} F_{1},
\end{aligned}
$$

where $S=\left\langle u_{1,1}^{3}\right\rangle /\left\langle u_{1,1}^{2}\right\rangle^{3 / 2}$ is the skewness of the longitudinal velocity gradient, which is a negative constant in HIT, and $K=\left\langle u_{1,1}^{4}\right\rangle /\left\langle u_{1,1}^{2}\right\rangle^{2}$ is the flatness of the longitudinal velocity gradient, which is related to the intermittency behavior.

Therefore, when $r \rightarrow 0$, substituting (14) in (13), finally we can obtain

$$
\begin{aligned}
F_{1}: F_{2}: F_{3}= & 1: \frac{2+2 G}{4+G}: \frac{4}{3} \\
& \times \frac{9(1+G)(5+G+2 H) K-2(4+G) H S^{2}}{(4+G)(8+3 G) K} .
\end{aligned}
$$

Also, the value of $F_{4}$ is independent of the other three values in MFT. If the pressure-term functions $G$ and $H$ tend to 0 when $r \rightarrow 0$, Eq. (15) is reduced to the RE state (7); if we choose $G=1 / 2$ and $H=6 K /\left(3 K-S^{2}\right)$ when $r \rightarrow 0$, Eq. (15) is reduced to the Gaussian state (4).

The Gaussian-RE line implies a linear interpolation between the Gaussian state and the RE state, leading to

$$
\frac{K}{S^{2}}=-\frac{16(4+G) H}{9\left(79 G+34 G^{2}-16 H-16 G H\right)},
$$

which implies a relation between the model functions $G$ and $H$ in MFT at small scales.

\section{Discussions on the fourth invariant $F_{4}$}

In the present contribution, the fourth invariant $F_{4}$ has not been discussed yet, simply because in the RE state $F_{4}$ is independent of the other three invariants and there remains only the Gaussian prediction for $F_{4}$. Here we attempt to discuss the behavior of $F_{4}$ from DNS evidence, though without much theoretical analysis. In Fig. 4 we plot all DNS results of Sec. III in the $\left(F_{2} / F_{1}, F_{3} / F_{1}\right)$ and $\left(F_{2} / F_{1}, F_{4} / F_{1}\right)$ planes, respectively. As already discussed, all $\left(F_{2} / F_{1}, F_{3} / F_{1}\right)$ points are located near the Gaussian-RE line, shown in Fig. 4(a). By contrast, the fitting line of all $\left(F_{2} / F_{1}, F_{4} / F_{1}\right)$ results in Fig. 4 (b) is far from the Gaussian state, which means that it is difficult to find

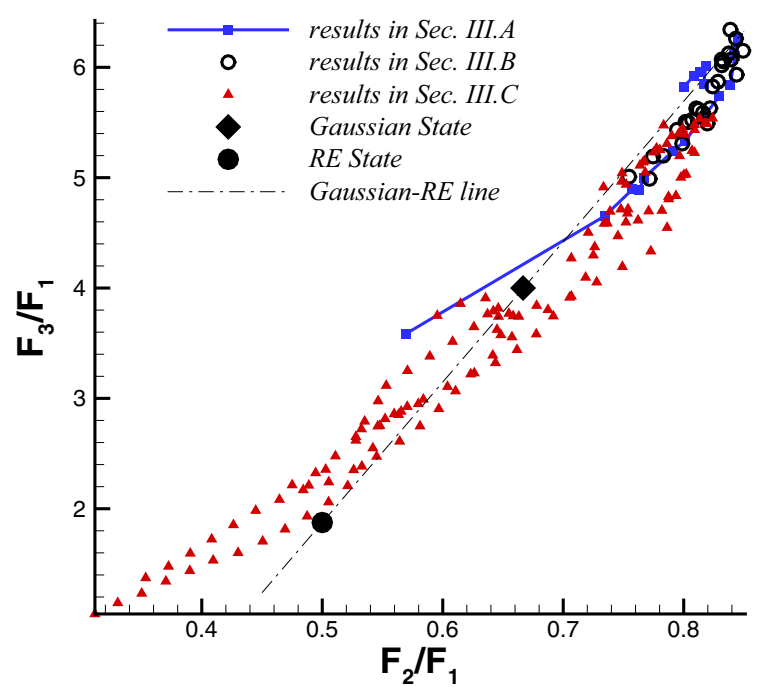

(a)

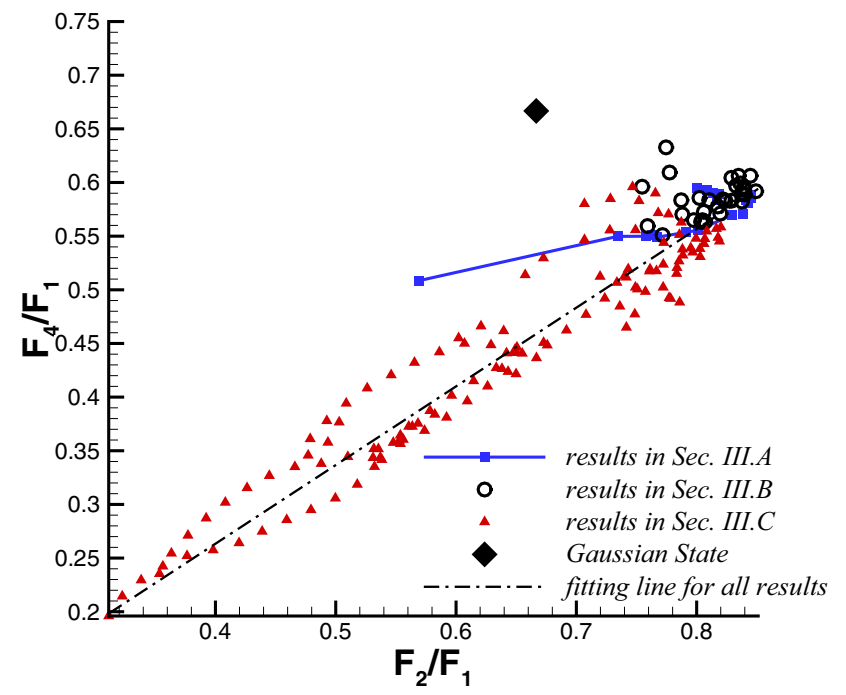

(b)

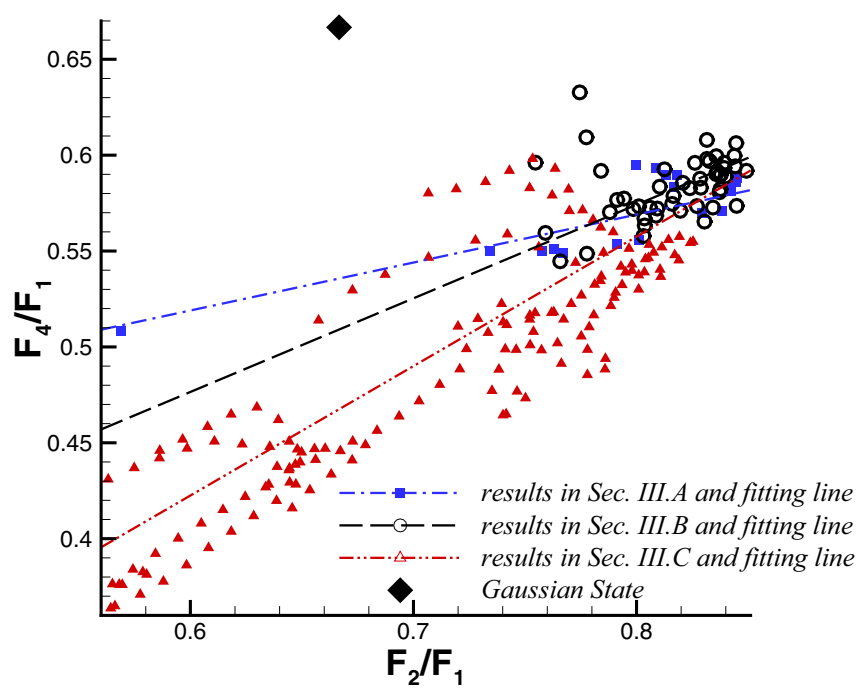

(c)

FIG. 4. Drawing all DNS results together. (a) The $\left(F_{2} / F_{1}, F_{3} / F_{1}\right)$ phase plane; (b) the $\left(F_{2} / F_{1}, F_{4} / F_{1}\right)$ phase plane; (c) the $\left(F_{2} / F_{1}, F_{4} / F_{1}\right)$ phase plane, zoomed view. 
another "Gaussian-RE" method to describe the behavior of $F_{4}$; moveover, the fitting lines for separated DNS groups are distinct, as shown in Fig. 4(c), implying that the physical laws (if they exist) for these DNS groups are not the same. Further investigations are therefore expected to clarify the physics of the fourth invariant $F_{4}$.

In addition, from Sec. IV A we argued that the Gaussian-RE line corresponds to a pressure effect that can be represented by a relation of the pressure-velocity correlations. Whether considering Eq. (9) or (12), there is only one equation for describing this pressure effect. Therefore, from the present analysis we can only obtain one pressure-caused constraint on the invariants (i.e., the Gaussian-RE line), and we cannot provide more linear constraints for describing $F_{4}$. We remark that the behavior of $F_{4}$ might be related to another physical mechanism, which is beyond the scope of the present contribution.

\section{CONCLUSION}

In the present contribution, we aim to investigate the relation of the fourth-order statistical invariants of a velocity gradient tensor in different types of isotropic turbulence. The observations are summarized and discussed as follows:

(i) All turbulent fields are located near the Gaussian-RE line in the $\left(F_{2} / F_{1}, F_{3} / F_{1}\right)$ phase plane, which implies that any turbulent field might be considered as, in the sense of fourth-order statistics, a superposition of the Gaussian state and the RE state. This fact, which corresponds to a pressure effect, reduces approximately one degree of freedom for the fourth-order tensor. This also leads to a new restriction on the MFT at small scales.

(ii) Each DNS run has its own "equilibrium region" that is roughly Reynolds-dependent. With a higher Reynolds number, the flow is more over-Gaussian in the $\left(F_{2} / F_{1}, F_{3} / F_{1}\right)$ phase plane.

(iii) Both the nonequilibrium transition process and the compressible transition process lead to the toward-equilibrium trajectories in the $\left(F_{2} / F_{1}, F_{3} / F_{1}\right)$ phase plane. We remark that compared to the recent studies on nonequilibrium turbulence based on energy transfer $[22,37,38]$, here the nonequilibrium is a description in the phase plane of fourth-order invariants, which may imply new physics on high-order statistics. In particular, the nonequilibrium evolution of compressible flows has not been reported in the literature, and future studies are called for.

(iv) There seems to be no universal law for the fourth invariant $F_{4}$ in the phase space. In particular, the Gaussian point is indeed far from all possible fitting lines for $F_{4}$.

We remark that the present contribution is not a comparison of the Gaussian and RE assumptions. Although most DNS cases are closer to the Gaussian state, we do not argue that the Gaussian assumption is better. Instead, we emphasize that both assumptions are appropriate simplifications that correctly capture parts of the physics, since both are located in the Gaussian-RE line, which is shown to be a good approximation for real turbulence. The present contribution also shows that both assumptions need to be relaxed to be more general for different turbulent flows: for the Gaussian assumption, one should consider the fourth-order accumulations [5,6] for relaxation; for the $\mathrm{RE}$ assumption, one should consider the pressure effect for obtaining more realistic models on the fourth-order moments. In future work, we therefore expect to investigate the possibility of decomposing a real flow field into a Gaussian field and an RE field (similar to the procedure in Ref. [22]) and to provide more realistic models.

Finally, we clarify that in the present contribution we use the term "over-Gaussian" to represent a behavior in the $\left(F_{2} / F_{1}, F_{3} / F_{1}\right)$ phase plane. It differs from the definition of "over-Gaussianity" in the research of intermittency, which implies that the flatness of velocity gradient $K$ is greater than the Gaussian value 3 and represents a strong intermittency.

\section{ACKNOWLEDGMENTS}

We are grateful to Wouter Bos for the important discussions and to the two anonymous referees for the many constructive suggestions. This work is supported by the National Science Foundation in China (Grants No. 11572025, No. 11202013, No. 11302012, and No. 51420105008) and the National Basic Research Program of China (Grant No. 2012CB720200).
[1] C. Meneveau, Lagrangian dynamics and models of the velocity gradient tensor in turbulent flows, Annu. Rev. Fluid Mech. 43, 219 (2011).

[2] M. Lesieur, Turbulence in Fluids (Kluwer Academic, Dordrecht, 1997).

[3] S. A. Orszag, Lectures on the statistical theory of turbulence: Fluid Dynamics, Les Houches Summer School of Theoretical Physics, edited by R. Balian and J. L. Peube (Gordon and Breach, New York, 1974), p. 677.

[4] R. H. Kraichnan, The structure of isotropic turbulence at very high Reynolds numbers, J. Fluid Mech. 5, 497 (1959).

[5] W. J. T. Bos and R. Rubinstein, On the strength of the nonlinearity in isotropic turbulence, J. Fluid Mech. 733, 158 (2013).

[6] W. Bos, R. Rubinstein, and L. Fang, Reduction of mean-square advection in turbulent passive scalar mixing, Phys. Fluids 24, 075104 (2012).
[7] J. O. Hinze, Turbulence, 2nd ed. (McGraw-Hill, New York, 1975).

[8] F. H. Champagne, The fine-scale structure of the turbulent velocity field, J. Fluid Mech. 86, 67 (1978).

[9] E. D. Siggia, Invariants for the one-point vorticity and strain rate correlation functions, Phys. Fluids 24, 1934 (1981).

[10] N. Phan-Thien and R. A. Antonia, Isotropic Cartesian tensors of arbitrary even orders and velocity correlation functions, Phys. Fluids 6, 3818 (1994).

[11] J. Hierro and C. Dopazo, Fourth-order statistical moments of the velocity gradient tensor in homogeneous, isotropic turbulence, Phys. Fluids 15, 3434 (2003).

[12] G. K. Batchelor, The Theory of Homogeneous Turbulence (Cambridge University Press, Cambridge, 1953).

[13] B. J. Cantwell, Exact solution of a restricted euler equation for the velocity gradient tensor, Phys. Fluids 4, 782 (1992). 
[14] R. J. Hill and O. N. Boratav, Next-order structure-function equations, Phys. Fluids 13, 276 (2001).

[15] B. J. Cantwell, On the behavior of velocity gradient tensor invariants in direct numerical simulations of turbulence, Phys. Fluids 5, 2008 (1993).

[16] J. Martin, A. Ooi, M. S. Chong, and J. Soria, Dynamics of the velocity gradient tensor invariants in isotropic turbulence, Phys. Fluids 10, 2336 (1998).

[17] Y. Li and C. Meneveau, Origin of Non-Gaussian Statistics in Hydrodynamic Turbulence, Phys. Rev. Lett. 95, 164502 (2005).

[18] B. Luthi, M. Holzner, and A. Tsinober, Expanding the QR space to three dimensions, J. Fluid Mech. 641, 497 (2009).

[19] T. Ishihara, Y. Kaneda, M. Yokokawa, K. Itakura, and A. Uno, Small-scale statistics in high-resolution direct numerical simulation of turbulence: Reynolds number dependence of one-point velocity gradient statistics, J. Fluid Mech. 592, 335 (2007).

[20] R. M. Kerr, Higher-order derivative correlations and the alignment of small-scale structure in isotropic numerical turbulence, J. Fluid Mech. 153, 31 (1985).

[21] L. Fang, W. J. T. Bos, L. Shao, and J.-P. Bertoglio, Timereversibility of Navier-Stokes turbulence and its implication for subgrid scale models, J. Turb. 13, 1 (2012).

[22] L. Fang, Y. Zhu, Y. W. Liu, and L. P. Lu, Spectral nonequilibrium property in homogeneous isotropic turbulence and its implication in subgrid-scale modeling, Phys. Lett. A 379, 2331 (2015).

[23] L. Fang, L. Shao, J. P. Bertoglio, G. Cui, C. Xu, and Z. Zhang, An improved velocity increment model based on Kolmogorov equation of filtered velocity, Phys. Fluids 21, 065108 (2009).

[24] J. Fang, Z. Li, and L. Lu, An optimized low-dissipation monotonicity-preserving scheme for numerical simulations of high-speed turbulent flows, J. Sci. Comput. 56, 67 (2013).

[25] S. K. Lele, Compact finite difference schemes with spectral-like resolution, J. Comput. Phys. 103, 16 (1992).

[26] S. Gottlieb and C. W. Shu, Total variation diminishing RungeKutta schemes, Math. Comput. 67, 73 (1998).
[27] Z. C. Qin, L. Fang, and J. Fang, How isotropic are turbulent flows generated by using periodic conditions in a cube? Phys. Lett. A 380, 1310 (2016).

[28] R. S. Rogallo, Numerical experiments in homogeneous turbulence, NASA Tech. Mem. 81315 (1981).

[29] M. Chertkov, A. Pumir, and B. I. Shraiman, Lagrangian tetrad dynamics and the phenomenology of turbulence, Phys. Fluids 11, 2394 (1999).

[30] A. Naso and A. Pumir, Scale dependence of the coarse-grained velocity derivative tensor structure in turbulence, Phys. Rev. E 72, 056318 (2005).

[31] L. Chevillard, C. Meneveau, L. Biferale, and F. Toschi, Modeling the pressure Hessian and viscous Laplacian in turbulence: Comparisons with direct numerical simulation and implications on velocity gradient dynamics, Phys. Fluids 20, 101504 (2008).

[32] M. Wilczek and C. Meneveau, Pressure Hessian and viscous contributions to velocity gradient statistics based on Gaussian random fields, J. Fluid Mech. 756, 191 (2014).

[33] L. Fang, W. J. T. Bos, and G. D. Jin, Short-time evolution of lagrangian velocity gradient correlations in isotropic turbulence, Phys. Fluids 27, 125102 (2015).

[34] P. K. Yeung, D. A. Donzis, and K. R. Sreenivasan, Dissipation, enstrophy and pressure statistics in turbulence simulations at high reynolds numbers, J. Fluid Mech. 700, 5 (2012).

[35] D. A. Donzis, P. K. Yeung, and K. R. Sreenivasan, Dissipation and enstrophy in isotropic turbulence: Resolution effects and scaling in direct numerical simulations, Phys. Fluids 20, 045108 (2008).

[36] S. Kurien and K. R. Sreenivasan, Dynamical equations for highorder structure functions, and a comparison of a mean-field theory with experiments in three-dimensional turbulence, Phys. Rev. E 64, 056302 (2001).

[37] P. C. Valente and J. C. Vassilicos, The non-equilibrium region of grid-generated decaying turbulence, J. Fluid Mech. 744, 5 (2014).

[38] P. C. Valente and J. C. Vassilicos, Universal Dissipation Scaling for Nonequilibrium Turbulence, Phys. Rev. Lett. 108, 214503 (2012). 